\title{
Nicotine coregulates multiple pathways involved in protein modification/degradation in rat brain
}

\author{
Justin K. Kane ${ }^{\mathrm{a}}$, Özlen Konu ${ }^{\mathrm{b}}$, Jennie Z. Ma ${ }^{\mathrm{a}}$, Ming D. Li ${ }^{\mathrm{a}, *}$

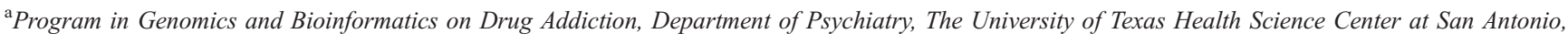 \\ San Antonio, TX 78229, United States \\ ${ }^{\mathrm{b}}$ Department of Molecular Biology and Genetics, Bilkent University, Ankara, Turkey
}

Accepted 12 September 2004

Available online 27 October 2004

\begin{abstract}
Previously, we used cDNA microarrays to demonstrate that the phosphatidylinositol and MAP kinase signaling pathways are regulated by nicotine in different rat brain regions. In the present report, we show that, after exposure to nicotine for 14 days, ubiquitin, ubiquitinconjugating enzymes, $20 \mathrm{~S}$ and $19 \mathrm{~S}$ proteasomal subunits, and chaperonin-containing TCP-1 protein (CCT) complex members are upregulated in rat prefrontal cortex (PFC) while being downregulated in the medial basal hypothalamus (MBH). In particular, relative to saline controls, ubiquitins B and $\mathrm{C}$ were upregulated by $33 \%$ and $47 \%(P<0.01)$, respectively, in the PFC. The proteasome beta subunit 1 (PSMB1) and 26S ATPase 3 (PSMC3) genes were upregulated in the PFC by $95 \%$ and $119 \%(P<0.001)$, respectively. In addition to the protein degradation pathway of the ubiquitin-proteasome complexes, we observed in the PFC an increase in the expression of small, ubiquitin-related modifiers (SUMO) 1 and 2 by $80 \%$ and $33 \%$, respectively $(P<0.001)$, and in 3 of 6 CCT subunits by up to $150 \%$ $(P<0.0001)$. To a lesser extent, a change in the opposite direction was obtained in the expression of the same gene families in the MBH. Quantitative real-time RT-PCR was used to validate the microarray results obtained with some representative genes involved in these pathways. Taken together, our results suggest that, in response to systemic nicotine administration, the ubiquitin-proteasome, SUMO, and chaperonin complexes provide an intricate control mechanism to maintain cellular homeostasis, possibly by regulating the composition and signaling of target neurons in a region-specific manner.
\end{abstract}

(C) 2004 Elsevier B.V. All rights reserved.

Theme: Neurotransmitters, modulators, transporters and receptors

Topic: Signal transduction, gene expression

Keywords: Nicotine; Ubiquitin; Proteasomes; cDNA microarrays; Real-time RT-PCR; Prefrontal cortex; Hypothalamus

\section{Introduction}

Nicotine is a potent agonist affecting various cellular processes throughout the brain. Acting on a diverse set of nicotinic acetylcholine receptors (nAChRs), nicotine can directly modulate the signaling pathways of a target neuron. Nicotine may also have indirect actions through influences on the release or uptake of neurotransmitters surrounding the parenchyma. The cellular and physiological consequences of nicotine's actions within the central nervous system (CNS)

\footnotetext{
* Corresponding author. Tel.: +210 567 0830; fax: +210 5670853

E-mail address: lim2@uthscsa.edu (M.D. Li).
}

are substantial and diverse. Although numerous in vitro and in vivo studies have been conducted to investigate how a gene of interest is modulated by nicotine, changes in gene expression profiles during acute and chronic exposure to the drug have been largely unknown.

The recent development of microarray technologies, allowing comparison of expression profiles of thousands of genes simultaneously, has revolutionized almost all biomedical research fields, including nicotine abuse research $[16,28,37]$. Previously, by using cDNA microarrays, we have shown that several genes such as rap 1 , Homer, and NF-kB and biochemical signaling pathways such as phosphatidylinositol and JNK cascades may play 
significant roles in nicotine dependence [28,37]. Using the same approach, in an accompanying paper [34], we followed the expression levels of 638 sequence-verified genes in five regions of the rat brain over time to gain a better understanding of how nicotine influences the molecular environment in physiologically pertinent brain regions.

Nicotine, a potent $\mathrm{nAChR}$ agonist, is likely to produce an altered environment within the CNS, forcing neurons to adapt. One mechanism used by the cell to sustain homeostasis under new environmental conditions is the ubiquitinproteasome pathway [21]. Ubiquitin is a conserved protein known to target proteins for degradation by the $26 \mathrm{~S}$ proteasome complex. Because of the specificity of ubiquitination, ubiquitin is responsible not only for targeting denatured proteins but also proteins that are intact but regulated in a time- or region-dependent manner. Through the destruction of these key signal proteins, the ubiquitin system is an important component of many signaling pathways [55]. For example, ubiquitin-mediated protein degradation controls cell cycle progression, signal transduction, transcriptional regulation, receptor downregulation, and endocytosis [12,13,21]. Moreover, under stress, proteins may become denatured and nonfunctional, producing an environment that may be cytotoxic. The ubiquitin/proteasomal system thus has a primary role in maintaining a healthy cellular environment by minimizing the accumulation of denatured and nonfunctional proteins. Secondarily, the system can play a key role in signaling pathways by targeting their rate-limiting proteins for destruction. An example of the impairment of the ubiquitin/proteasome system by a drug of abuse is ethanol's ability to partially inactivate the proteasome and block the ubiquitination of protein targets $[7,15]$.

Whereas the ubiquitin/proteasome pathway targets proteins for destruction, chaperones are involved in the folding of newly synthesized proteins as well as refolding of proteins denatured under environmental stress. The chaperonin-containing TCP-1 (CCT) is a subclass of the chaperonin protein family that aids in the folding of large nonnative proteins [19,31]. For example, CCT has a hetero-oligomeric structure that helps to create the correct 3-D structure of cytoskeletal proteins such as actin, tubulin and other polypeptides in the cytosol [51]. Because of its constructive functionality, CCT is likely to work in concert with other protein modification and degradation pathways for the plasticity and maintenance of cellular homeostasis.

These multiple pathways are known to crosstalk and are the product of interconnected gene networks. Our previous investigations have demonstrated the value of microarrays in tracking nicotine-induced changes in expression of genes that belong to the phosphatidylinositol-driven pathways, both in the brain and in PC-12 cells [28,37]. These initial findings implicated nicotine as an important modulator of cellular homeostasis, thus warranting its further study in relation to other homeostatic pathways such as those of protein modification and degradation. In this study, we focused on investigating the correlation of mRNA transcription profiles of protein modification/degradation complexes with subacute and chronic exposure to nicotine (i.e., 3 and 14 days) within the prefrontal cortex (PFC) and the medial basal hypothalamus (MBH). Our results revealed that ubiquitin-proteasome, SUMO, and CCT complex members contribute to the changes induced by nicotine in a region- and time-specific manner. We present the first report of the involvement of multiple yet interconnected cellular pathways of protein modification and degradation in response to chronic administration of nicotine in rat $\mathrm{PFC}$ and $\mathrm{MBH}$.

\section{Materials and methods}

\subsection{Animals, nicotine administration, and brain punches}

Male Holtzman rats (250-350 g; HSD, Madison, WI) received either nicotine dihydrochloride at a dose of $4.0 \mathrm{mg} /$ $\mathrm{kg}$ /day in saline ( $\mathrm{pH} 7.4)$ or saline alone by intraperitoneal (ip) injection. The rats quickly became tolerant to the dose, and no seizures were observed $[24,36]$. An initiation phase of $48 \mathrm{~h}$ was used to habituate the animals to the stress of the injection, as confirmed by reduction of c-fos expression [47]. Rats were maintained on a 12:12 h light/dark cycle with standard laboratory rat chow and water freely available.

After the conclusion of nicotine administration, rats were injected with a lethal overdose of sodium pentobarbital (100 $\mathrm{mg} / \mathrm{ml}$; ip) and decapitated, and their brains were removed. Coronal 2-mm sections were prepared, and bilateral specimens were obtained from the PFC and $\mathrm{MBH}$ using a brain punch tissue set from myNeuroLab.com (St. Louis, MO) at sites chosen according to the coordinates from Paxinos and Watson [45].

\subsection{Total RNA isolation and amplification}

Total RNA was isolated using TRIzol reagent according to the manufacturer's protocol (Qiagen, Valencia, CA). Before use, RNA samples were treated with RNAse-free DNAse I at $37{ }^{\circ} \mathrm{C}$ for $30 \mathrm{~min}$. The integrity of the samples was assessed by viewing the ethidium bromide-stained $28 \mathrm{~S}$ and $18 \mathrm{~S}$ ribosomal RNA bands. A T7 amplification protocol was used to produce the amount of starting RNA required for the synthesis of fluorescent cDNA probes [54].

\subsection{Microarray production, $c D N A$ probe labeling, and array hybridization}

A pathway-focused microarray consisting of 638 sequence-verified genes that was designed by our laboratory (called the homeostatic pathway-focused array) was used [52]. This array contains 638 sequence-verified genes selected from rat cDNA clones (Research Genetics, AL) and the 15K NIA mouse cDNA gene set [52] on the basis of our previous studies $[28,37]$. It was shown that cross-species 
hybridization (i.e., mouse clones against rat target samples) using a cDNA platform is accurate and specific [59]. Cells containing the cDNA clones of interest were grown overnight, and $10 \mu \mathrm{l}$ of the culture medium plus $90 \mu \mathrm{l}$ of water were incubated at $95^{\circ} \mathrm{C}$ for $10 \mathrm{~min}$ to release plasmid DNA. A $10-\mu 1$ sample of supernatant liquid was added to a PCR cocktail mixture containing Taq polymerase in a total volume of $100 \mu \mathrm{l}$. The reaction mixtures were denatured at $95{ }^{\circ} \mathrm{C}$ for $3 \mathrm{~min}$ and then subjected to 35 cycles of denaturation, annealing, and extension. The products were ethanol precipitated, washed and reconstituted in TE buffer. To increase the reliability of the measurements, $30 \mu \mathrm{l}$ of $80 \%$ dimethyl sulfoxide (DMSO; Sigma) was added to the dissolved PCR products. The slides were printed by the GMS 417 arrayer (Affymetrix, Santa Clara, CA) according to the protocols suggested by the manufacturer. cDNA probe labeling and array hybridization were performed as described previously with slight modifications [28,29].

\subsection{Microarray data normalization and statistical analysis}

The procedures used for normalization and detection of significantly expressed genes were described in detail previously. For any given time point of treatment, we started the experiments with six control and six nicotinetreated animals. However, for some regions, the final number of RNA samples or microarray hybridizations/slides was less because we lost some samples during the animal experiments, did not have enough RNA for probe labeling, or eliminated slides because the overall duplicate spot correlation coefficient value was $<0.9$. Ultimately, the PFC had three controls and two nicotine-treated animals at day 3 and three control and three nicotine-treated animals at day 14, while the $\mathrm{MBH}$ had three control and five nicotinetreated animals at day 3 and five control and five nicotinetreated animals at day 14. Given that each gene was printed in duplicate on the array, expression of the genes reported was derived from at least four independent measurements. Analysis of variance was used to test the significance of differentially expressed genes. All these processes involving data normalization and analysis were performed in programs written in MatLab (Mathworks, Natick, MA) or SAS (SAS Institute, Cary, NC).

\subsection{Validation of microarray results by real-time quantita- tive RT-PCR}

Microarray results were validated with real-time quantitative RT-PCR using a strategy described previously [35] with modifications $[8,28]$. After the last cycle, a dissociation curve was run to check for product purity. The expression level of each gene was determined after specifying an amplification cycle corresponding to the exponential growth phase of the RT-PCR reaction for that gene. Intensity values based on the incorporation of fluorescent SYBR ${ }^{\circledR}$ Green dye (excitation/emission $497 / 520 \mathrm{~nm}$ ) into the double-

Table 1

Summary of selected genes and their primer information used in quantitative real-time RT-PCR amplification

\begin{tabular}{|c|c|c|c|}
\hline Gene name & Abbreviation & Accession no. & Primer sequence $\left(5^{\prime} \rightarrow 3^{\prime}\right)$ \\
\hline Proteasome subunit, beta Type 1 & Psmb1 & ВC018351 & $\begin{array}{l}\text { P1:GCA TCA GGG AGG AGA CTG TT } \\
\text { P2:TAA AAC AAA CGT GCC ACA GT }\end{array}$ \\
\hline Proteasome subunit, beta Type 3 & Psmb3 & BC014783 & $\begin{array}{l}\text { P1:GCG TCA TTG TCC ACA TCA TT } \\
\text { P2:GCC AGA GCA CAG GTT AGT CC }\end{array}$ \\
\hline Proteasome subunit, beta Type 2 & Psmb2 & AF060090 & $\begin{array}{l}\text { P1:CCC CCA CAG CAG CAG CTA AT } \\
\text { P2:AGC CAG CCA GGA GGA GGT TC }\end{array}$ \\
\hline Proteasome ATPase p45/SUG & SUG1 & AB000491 & $\begin{array}{l}\text { P1:AAC CAG CTG GAT GGC TTT GA } \\
\text { P2:GCA GGG CAG AGT CCA GGA TA }\end{array}$ \\
\hline Proteasome $26 \mathrm{~S}$ subunit, ATPase 3 & Psmc3 & NM_031595 & $\begin{array}{l}\text { P1:GAC CTG GAC TCG CAG AGG AA } \\
\text { P2:AAC CCA ATC ACT GGC AGG AA }\end{array}$ \\
\hline Proteasome subunit 26S, non-ATPase 4 & Psmd4 & NM_031331 & $\begin{array}{l}\text { P1:CAG GAC CCA GAG TTC CTT CA } \\
\text { P2:ATT GCA TTT CGA ATG GCT TC }\end{array}$ \\
\hline Proteasome 26S subunit, non-ATPase 13 & Psmd13 & NM_011875 & $\begin{array}{l}\text { P1:GTT TGG CAC CGT TTG GAA GA } \\
\text { P2:GCA TGG GTC CTG CAC AAA AT }\end{array}$ \\
\hline Rat ubiquitin B-like & $\mathrm{rUbb}$ & D16554 & $\begin{array}{l}\text { P1:TGA GGA CTG CAG CCA ACA CC } \\
\text { P2:GGT GTC ACT GGG CTC GAC CT }\end{array}$ \\
\hline Ubiquitin-conjugating enzyme 5 & Ube $2 \mathrm{e} 1$ & ВC003781 & $\begin{array}{l}\text { P1:GGC CAG ACA GTG GAC CAA GA } \\
\text { P2:CAC CCC CAC CCT TTC AAA AT }\end{array}$ \\
\hline Ubiquitin-like protein 1 & SUMO-1 & NM_009460 & $\begin{array}{l}\text { P1:CCA GGA GGC AAA ACC TTC AAC } \\
\text { P2:CAC TGC TAT CCT GTC CAA TGA C }\end{array}$ \\
\hline Ubiquitin-like protein 2 & SUMO-2 & NM_133594 & $\begin{array}{l}\text { P1:GGG ACA GGA TGG TTC TGT GG } \\
\text { P2:CAA ACC CTG CCG TTC ACA AT }\end{array}$ \\
\hline Ubiquitin-conjugating enzyme E2I & Ube2I & NM_013050 & $\begin{array}{l}\text { P1:GAC TTG CGC AGG AGA GGA AA } \\
\text { P2:TCG TGC CAT CAG GGT TCT TT }\end{array}$ \\
\hline Chaperonin subunit 3 (gamma) & CCT3 & Z31556 & $\begin{array}{l}\text { P1:AGA TGC TGG CCA GGA GTG AG } \\
\text { P2:TCC TGG AAC TCT GGC TCA GG }\end{array}$ \\
\hline Chaperonin subunit 7 (beta) & CCT7 & Z31399 & $\begin{array}{l}\text { P1:GGA CAT TGC TGA CAA CTT CC } \\
\text { P2:CAG GCA CGC AGC CTC AGA AG }\end{array}$ \\
\hline
\end{tabular}


stranded PCR products for control and treatment samples were measured at these specified amplification cycles. All genes were normalized to the 18S rRNA level. The primer sequences used for PCR amplification in conjunction with other related information are summarized for each gene in Table 1.

All of the techniques used are described in detail elsewhere (Refs. [28,29,34]).

\section{Results}

\subsection{Upregulation of ubiquitin and SUMO gene families in the PFC after 14 days of nicotine administration}

In the rat, two main transcripts encode ubiquitins B and C $[18,20]$. Our pathway-focused microarray contains three ubiquitin-derived clones; two are mouse ubiquitins B and $\mathrm{C}$, and the third is a rat ubiquitin homologous to mouse ubiquitin $\mathrm{B}$. In the $\mathrm{PFC}$, we observed an increase in mRNA expression for mouse ubiquitins $\mathrm{B}$ and $\mathrm{C}$ and the rat ubiquitin $\mathrm{B}$ after 14 days of nicotine administration (33\%, 47\%, 38\%, respectively; $P<0.01$ for all of them; Table 2).

In addition to ubiquitin expression, upregulation of ubiquitin-conjugating E2 mRNAs was observed. For example, we found that nicotine treatment increased ubiquitinconjugating enzyme E2I (Ube2I) expression by $37 \%$ $(P<0.01$; Table 2$)$ and ubiquitin protein ligase E3B (Ube3b) expression by $21 \%(P=0.08)$ in the PFC. Except for a modest increase in ubiquitin fusion degradation 1 at day 3 $(12 \% ; P<0.05)$, the ubiquitin gene family did not show a significant response to nicotine in the PFC after 3 days of treatment.

While SUMO is activated and targets substrate proteins in a fashion analogous to ubiquitin, the fate of the substrate being sumoylated is different. SUMO-1 modulates the interaction of the target protein with other cellular proteins and often results in changing the protein's location $[9,27,43]$. For example, a SUMO-attached protein may be transported to the nucleus [27,39]. Furthermore, there exist functional differences between SUMO-1, SUMO-2 and SUMO-3, such that a greater abundance of SUMO-2 and SUMO-3 acts as a stress response compared with SUMO-1 [48]. After 14 days of nicotine administration, both SUMO1 and SUMO-2 were modulated with an increase in expression by $79 \%(P<0.0001)$ and $33 \%(P<0.001$; Fig. $1)$, respectively. These findings have been confirmed by quantitative real-time RT-PCR (see Fig. 5 below). Furthermore, expression of Ube2I/Ubc9, the only E2 enzyme of sumoylation, was significantly (37\%) upregulated in the PFC $(P<0.001$; Table 2).

\subsection{Upregulation of proteasome-complex genes in the PFC after 14 days of nicotine treatment}

Once a protein has been ubiquitinated, it is brought to the 26S proteasome containing the $20 \mathrm{~S}$ catalytic core and two 19S regulatory complexes for degradation. The mammalian 20S proteasome is made up of seven different alpha and seven different beta subunits, with the beta subunits comprising the inner rings, where the actual cleavage of proteins occurs. Our array results demonstrated an increase in proteasome subunits: beta type 1 after $3(39 \% ; P<0.05)$ and $14(95 \% ; P<0.0001)$ days of nicotine administration and beta type $2(21 \% ; P=0.01)$ and beta type $5(33 \%$; $P<0.05$; see Table 3) after 14 days.

The 19S complex is made up of different ATPase and non-ATPase subunits that aid in regulating the 20S complex. On day 14, we found an increase in the ATPase subunit 3 $(119 \% ; P<0.0001)$ and ATPase subunit SUG1/P45 (39\%; $P<0.05$; see Table 3). Significant increases in non-ATPase subunits $4(60 \% ; P<0.01)$ and $13(94 \% ; P<0.0001)$ also were detected on day 14 .

Table 2

Expression of ubiquitin and SUMO-related protein genes in PFC at 3 and 14 days of nicotine administration, as determined by microarray analysis

\begin{tabular}{|c|c|c|c|c|}
\hline Gene name & Abbreviation & Accession no. & $\begin{array}{l}\text { Day } 3 \text { mRNA } \\
(\% \text { change } \pm \text { S.E. })\end{array}$ & $\begin{array}{l}\text { Day } 14 \text { mRNA } \\
(\% \text { change } \pm \text { S.E. })\end{array}$ \\
\hline Ubiquitin fusion degradation 1 & Ufd11 & U64445 & $12.0 \pm 3.9 *$ & $152.3 \pm 12.1 * * *$ \\
\hline Ubiquitin-specific protease 5 & Usp5 & NM_013700 & $-5.0 \pm 2.6$ & $87.2 \pm 5.4 * * *$ \\
\hline Ubiquitin-like 1 & SUMO-1 & NM_009460 & $2.8 \pm 13.4$ & $79.1 \pm 7.1 * * *$ \\
\hline Ubiquitin $\mathrm{C}$ & Ubc & AF285162 & $70.5 \pm 32.3$ & $47.0 \pm 10.7 * *$ \\
\hline Ubiquitin specific protease 8 & Ubp8 & AF057146 & $-12.0 \pm 6.9$ & $39.6 \pm 6.6^{* * *}$ \\
\hline Ubiquitin A-52 residue ribosomal protein fusion product 1 & Uba52 & NM_031687 & $60.4 \pm 31.1$ & $39.4 \pm 4.7 * * *$ \\
\hline Rat ubiquitin B-like & $\mathrm{rUbb}$ & D16554 & $16.5 \pm 8.7$ & $37.5 \pm 8.3 * *$ \\
\hline Ubiquitin-conjugating enzyme E2I & Ube2I & NM_013050 & $2.2 \pm 4.7$ & $36.5 \pm 8.5 * *$ \\
\hline Ubiquitin-like 2 & SUMO-2 & NM_133594 & $1.8 \pm 15.5$ & $33.4 \pm 5.6^{* *}$ \\
\hline Ubiquitin B & Ubb & NM_011664 & $27.6 \pm 21.2$ & $33.2 \pm 8.2 * *$ \\
\hline Ubiquitination factor E4B & Ube $4 b$ & AF260926 & $-6.0 \pm 6.4$ & $31.5 \pm 5.3 *$ \\
\hline Ubiquitin-specific protease & Usp2 & NM_016808 & $-7.7 \pm 12.2$ & $12.6 \pm 3.2 *$ \\
\hline Ubiquitin-conjugating enzyme 5 & Ube2e1 & ВC003781 & $-3.9 \pm 13.6$ & $-23.8 \pm 2.4 * * *$ \\
\hline
\end{tabular}

$* P<0.05$.

** $P<0.01$.

*** $P<0.001$. 
A)

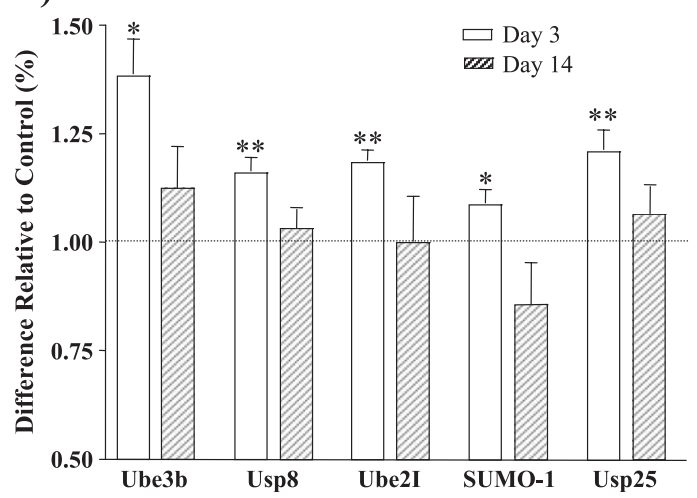

B)

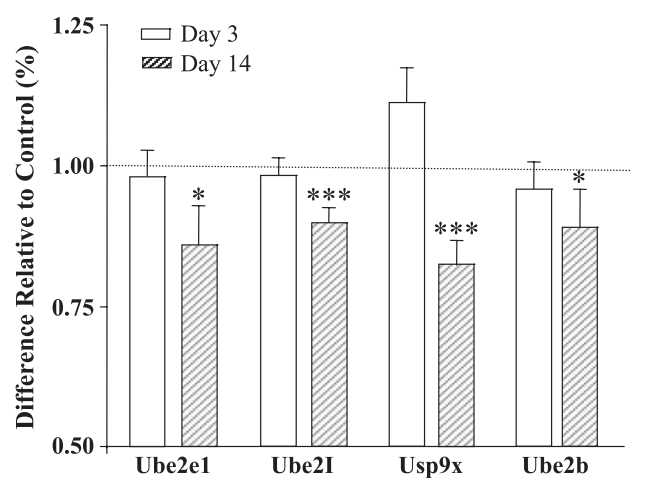

Fig. 1. Expression of ubiquitin-related genes in MBH at 3 and 14 days of nicotine administration, as determined by microarray analysis. (A) Expression of ubiquitin-related genes is upregulated significantly at 3 days. (B) Expression of genes of several ubiquitin-conjugating enzymes of this family is significantly downregulated at 14 days. Abbreviations: ubiquitin protein ligase E3B (Ube3b, BC034059); ubiquitin-specific protease 8 (Usp8, BC027052); ubiquitinconjugating enzyme E2I (Ube2I, NM_013050); ubiquitin-like 1 (SUMO-1, NM_009460); ubiquitin-specific protease 25 (Usp25, AF170563); ubiquitinconjugating enzyme 5 (Ube2e1, BC003781); ubiquitin-specific protease 9 (Usp9x, NM_009481), ubiquitin-conjugating enzyme E2b (Ube2b, AF144083). Significance level: $* P<0.05, * * P<0.01, * * * P<0.001$.

\subsection{Expression of proteasomal and ubiquitin genes in the MBH decreases over 14 days of nicotine administration}

The MBH is a structurally diverse brain region involved in a variety of physiological functions. While we observed a transient increase in expression of the ubiquitin family genes in the MBH on day 3 (Fig. 1A), this increase was followed by a significant decline in expression of several ubiquitinconjugating enzymes on day 14 (Fig. 1B). Indeed, proteasome subunits alpha $2(15 \% ; P<0.01), 3(15 \%$; $P<0.01)$ and $6(8 \% ; P<0.01)$ were all downregulated after 14 days of nicotine treatment (Fig. 2), contrasting with the pattern we observed for the set of proteasome beta subunits in the PFC at the same time point.

\subsection{Chaperonin subunits are upregulated in the PFC but downregulated in the MBH after 14 days of nicotine treatment}

There are many families of chaperones (e.g., heat shock proteins; for a review, see Ref. [19]). Our pathway-focused array contains sequences encoding five of the eight subunits that make up the chaperonin-containing TCP-1 protein (CCT). Chaperonin subunits 3 (120\%; $P<0.0001)$, 7 (158\%; $P<0.0001)$, and $8(70 \% ; P<0.0001)$ were upregulated in the $\mathrm{PFC}$ at 14 days of nicotine treatment (Fig. 3). In contrast, chaperonin subunits $2(16 \% ; P<0.01), 3(12 \% ; P<0.01), 4$ $(16 \% ; P<0.05)$, and $8(22 \% ; P<0.001)$ were downregulated in the MBH (Fig. 4). No significant differences were observed in the chaperonin gene family expression in the $\mathrm{PFC}$ or MBH after 3 days of nicotine administration.

\subsection{Quantitative real-time RT-PCR confirms a majority of genes identified by microarray analysis}

The microarray results were validated using quantitative real-time RT-PCR with representative gene-specific primers on RNA samples extracted from an independent animal experiment. Primer sequences were selected according to the cDNA sequence printed on the microarray (see Table 1 for a detail). Fig. 5 shows side-by-side comparisons of realtime RT-PCR and microarray data of nine genes in the PFC

Table 3

Expression of proteasome-related protein genes in PFC at 3 and 14 days of nicotine administration, as determined by microarray analysis

\begin{tabular}{lllcc}
\hline Gene name & Abbreviation & Accession no. & $\begin{array}{l}\text { Day 3 mRNA } \\
\text { (\% change } \pm \text { S.E.) }\end{array}$ & $\begin{array}{l}\text { Day 14 mRNA } \\
\text { (\% change } \pm \text { S.E.) }\end{array}$ \\
\hline Proteasome 26S, ATPase 3 & Psmc3 & NM_031595 & $1.1 \pm 16.4$ & $119.1 \pm 8.5^{* * *}$ \\
Proteasome 26S, non-ATPase, 13 & Psmd13 & NM_011875 & $-4.1 \pm 5.0$ & $94.0 \pm 7.6^{* * *}$ \\
Proteasome subunit, beta type 1 & Psmb1 & BC018351 & $39.3 \pm 5.8^{*}$ & $95.4 \pm 7.7 * * *$ \\
Proteasome 26S, non-ATPase, 4 & Psmd4 & NM_031331 & $-7.1 \pm 6.7$ & $60.2 \pm 4.0^{* *}$ \\
Proteasome subunit, beta type 5 & Psmb5 & AF060091 & $10.7 \pm 20.1$ & $33.0 \pm 5.3 *$ \\
Proteasome ATPase p45/SUG & SUG1 & AB000491 & $7.6 \pm 8.9$ & $38.6 \pm 12.4^{*}$ \\
Proteasome 26S, ATPase 5 & Psmc5 & BC030840 & $-1.2 \pm 5.4$ & $27.5 \pm 2.4^{* * *}$ \\
Proteasome subunit, beta type 2 & Psmb2 & AF060090 & $-4.8 \pm 2.7$ & $20.5 \pm 4.8^{*}$ \\
\hline
\end{tabular}

* $P<0.05$

** $P<0.01$.

*** $P<0.001$ 


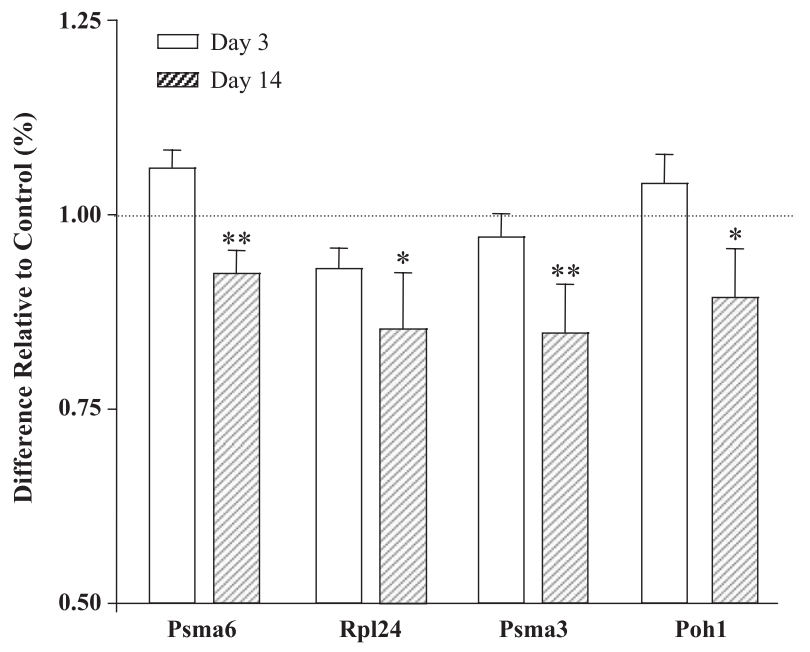

Fig. 2. Expression of genes for several alpha subunits from $20 \mathrm{~S}$ proteasomal complex in MBH at 3 and 14 days of nicotine administration, as determined by microarray analysis. Expression of most of these alpha subunits is downregulated at 14 days. No significant differences were obtained at 3 days. Abbreviations: proteasome subunit, alpha 6 (Psma6, U60288); proteasome subunit, alpha 2 (Psma2, NM_008944); proteasome subunit, alpha 3 (Psma3, AF055983); proteasome-associated pad1 (Poh1, BC003742). Significance level: $* P<0.05, * * P<0.01$.

after 14 days of nicotine administration. The real-time RTPCR confirmed the significant increases in the expression of rat polyubiquitin $(26 \% ; P<0.05)$, SUMO-1 $(20 \% ; P=0.08)$ and SUMO-2 (37\%; $P<0.05$; Fig. 5A). The real-time RTPCR results for the selected CCT components (Fig. 5B) showed $20 \%$ and $25 \%(P<0.05)$ increases in subunits CCT3 and $\mathrm{CCT} 7$, respectively. For the different proteasome subunits, the RT-PCR confirmed the increase in the beta type 1 subunit $(46 \% ; P<0.05), 26 \mathrm{~S}$ subunit ATPase $3(39 \%$;

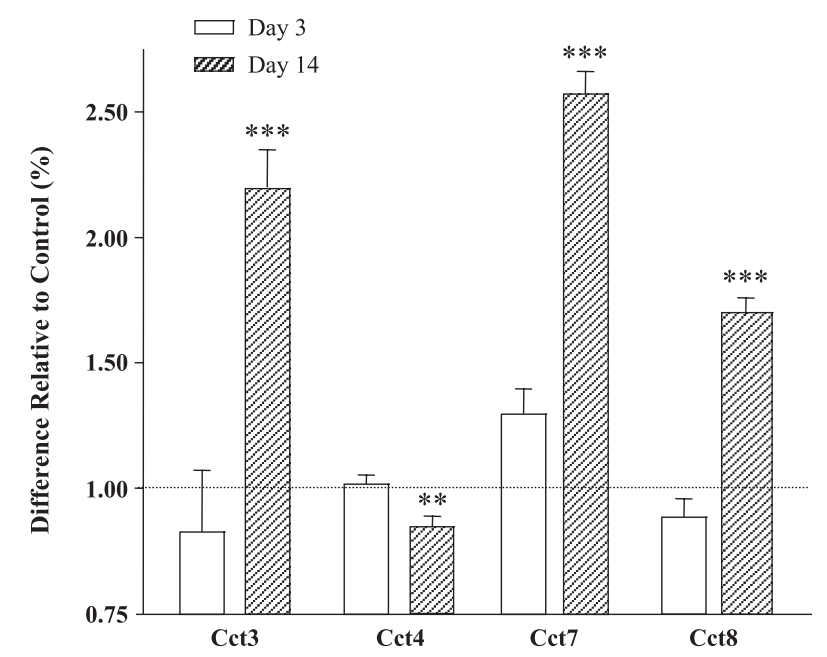

Fig. 3. Expression of chaperonin-related protein genes in PFC at 3 and 14 days of nicotine administration, as determined by microarray analysis. Significant changes were obtained for subunits 3, 4, 7, and 8 at 14 days. Abbreviations: chaperonin subunit 3 (CCT3, Z31556); chaperonin subunit 4 (CCT4, NM_009837); chaperonin subunit 7 (CCT7, Z31399) and chaperonin subunit 8 (CCT8, Z37164). Significance level: $* P<0.05$, ${ }^{* *} P<0.01,{ }^{* * *} P<0.001$.

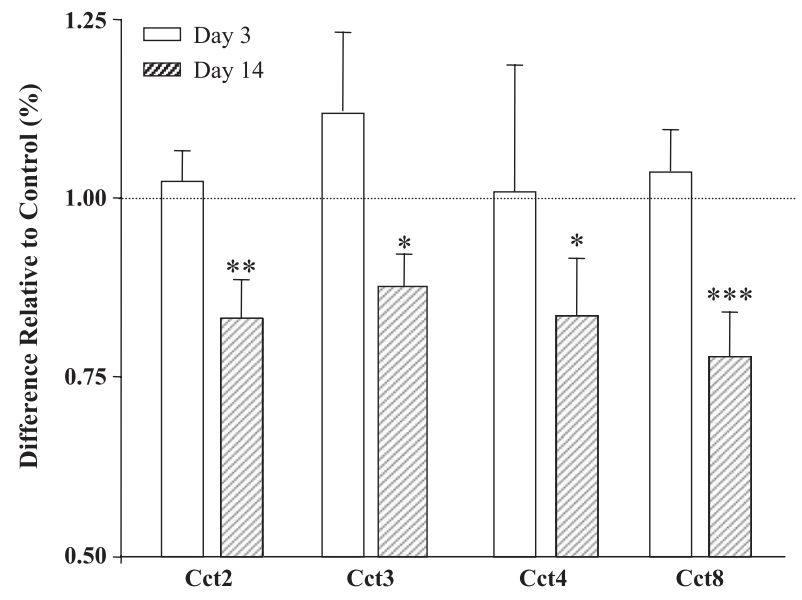

Fig. 4. Expression of chaperonin-related protein genes in $\mathrm{MBH}$ at 3 and 14 days of nicotine administration $\left({ }^{*} P<0.05\right)$. The CCT subunits $2,3,4$, and 8 are downregulated by approximately $12 \%$ to $22 \%$. While downregulation is modest (possibly because of diluting effects of a diverse structure), the nicotine effect on four subunits (with seven of the eight subunit genes printed on the array) indicates a stronger correlation for downregulation of this complex. Significance level: $* P<0.05,{ }^{*} P<0.01,{ }^{* * *} P<0.001$.

$P<0.05)$, and 26S subunit non-ATPase $13(27 \% ; P<0.05)$ and demonstrated an upward trend for the SUG1 gene (22\%; $P=0.07$; Fig. 5C).

\section{Discussion}

In addition to identifying candidate genes of interest for further molecular characterization such as traditional library screening or differential display, chip technology has another powerful application; i.e., to investigate how a group of function-related genes within a biochemical pathway respond to a treatment. One of the primary purposes of this communication is to illustrate such an application of microarrays in nicotine research. Protein synthesis and degradation are dynamic processes controlled by numerous factors and at different levels. For example, a cell may utilize the ubiquitin-proteasomal pathway to control the duration that a protein remains intact. As a result of its widespread utility, the ubiquitin-proteasomal pathway is complex, with many proteins required to work in concert. By using the pathway-focused microarray developed in this laboratory, we found a number of genes from the ubiquitin-proteasome and SUMO pathways, as well as the CCT gene family, that are highly regulated by chronic nicotine exposure in the rat PFC and $\mathrm{MBH}$.

Our pathway-focused microarray analyses indicated an increase in the expression of several members of the ubiquitin and proteasome gene families in the PFC with nicotine administration. In the PFC, both major ubiquitin $\mathrm{B}$ and $\mathrm{C}$ transcripts were upregulated at day 14 . In addition, the expression levels of ubiquitin-conjugating enzymes, ubiquitin-specific proteases, and deubiquitinating enzymes were upregulated, suggesting a general activation of the 
(A)

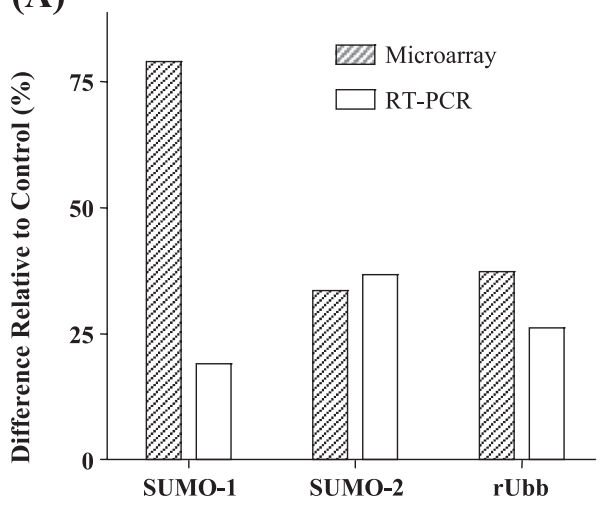

C)

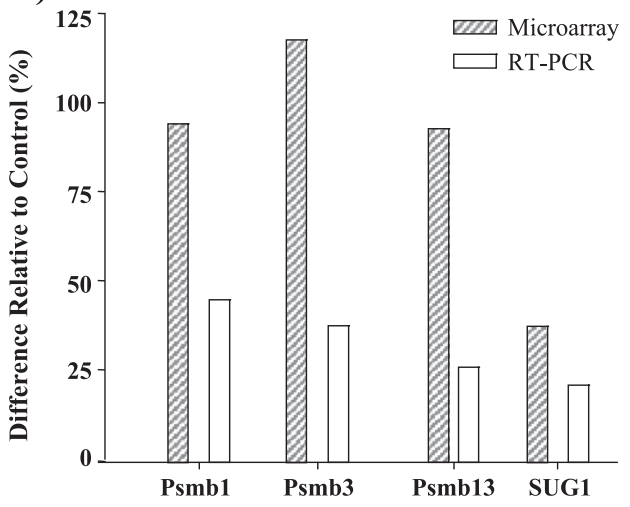

(B)

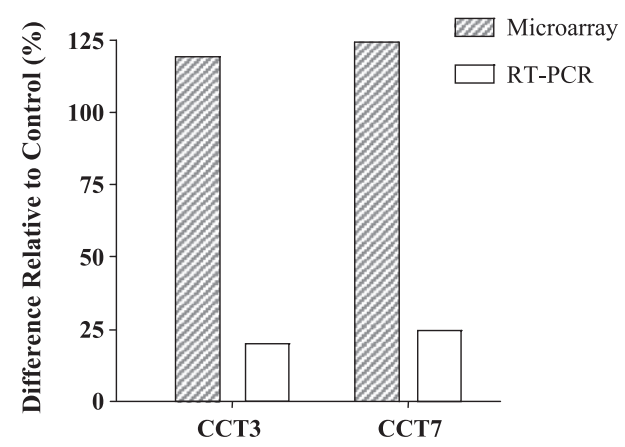

Fig. 5. Comparison of microarray and quantitative real-time RT-PCR results of selected genes in PFC after 14 days of nicotine administration. (A) Expression of ubiquitin and ubiquitin-related genes. (B) Levels of chaperonin subunits. (C) RNA levels of proteasomal-related genes. Verification of selected genes by real-time RT-PCR was performed in an independent animal experiment. For abbreviations, see Table 1.

ubiquitin system by nicotine in the PFC region. Complementing the increase in the ubiquitin system, there was an increase in expression of $20 \mathrm{~S}$ proteasome subunits and of regulatory subunits of the $19 \mathrm{~S}$ complex. The expression levels of two of the six ATPase subunits from the 19S base complex were upregulated as well, possibly indicating an increase in the need to provide the energy required in forming the $26 \mathrm{~S}$ proteasome.

In contrast to the PFC region, in the $\mathrm{MBH}$, members of the ubiquitin-proteasome gene families were downregulated after 14 days of nicotine administration. Three alpha subunits of the $20 \mathrm{~S}$ complex were downregulated by approximately $10 \%$ to $15 \%$. While no ubiquitin or SUMO genes were differentially regulated at 14 days, expression of two ubiquitin-conjugating and one SUMO-conjugating enzymes was decreased. Such differential responses to nicotine support the concept that various brain regions participate in different cellular and physiological functions. Indeed, a gene expression profiling study demonstrated similar profiles in subnuclei of the same brain region (i.e., $\mathrm{Ca} 1$ and $\mathrm{Ca} 3$ of the hippocampus) but very different profiles in different brain regions (i.e., hypothalamus and hippocampus) [6]. Because different regions of the brain are divergent in their cellular compositions (i.e., neurotransmitter release, receptor expression, intracellular signaling cascades) to manage specific physiological activities, a region-specific transcriptional response of ubiquitin-proteasome signaling to nicotine treatment would be expected.

The ubiquitin-proteasome pathway is responsible for the majority of intracellular protein degradation. Thus, it functions in a variety of capacities, all of which aim to produce protein homeostasis under different conditions. While the ubiquitin-proteasome pathway is constitutively involved in protein degradation, there are several known activation signals leading to its upregulation. For example, environmental and oxidative stresses that damage proteins activate the transcription of ubiquitin by increasing ubiquitin $\mathrm{B}$ and $\mathrm{C}$ transcripts [18] and converting free ubiquitin to a conjugated pool $[11,21]$. Although drugs of abuse, such as alcohol, nicotine and cocaine, induce oxidative damage and, hence, cellular stress $[4,10,57]$, the involvement of the ubiquitin-proteasome pathway in the response to nicotine treatment has remained largely unexplored. Because nicotine produces oxidative stress, protein denaturation and aggregation may be underlying effects the cell must combat $[56,57]$. Thus, protein modification/degradation pathways may be forced to respond to the changing conditions of the cell to produce homeostatic levels of proteins (see Fig. 6 for a proposed model). Furthermore, the observation that the CCT and ubiquitin-proteasome gene families are both 
upregulated in the PFC but downregulated in the $\mathrm{MBH}$ points to a common response element for the two gene families in these brain regions responding to the altered cellular environment brought about by nicotine.

Another pertinent role for the ubiquitin/proteasomal system has been demonstrated by Ehlers [17], who showed the regulation of numerous postsynaptic proteins by ubiquitination in response to synaptic activity in cultured rat hippocampal neurons. Recently, the ubiquitination process was linked to the regulation of the assembly and cell-surface trafficking of nAChRs $[25,26]$. We propose that nicotine increases synaptic activity while simultaneously producing a state of oxidative stress within certain areas of the brain. This, in turn, may lead to the modulation of the ubiquitin-proteasome pathway in an effort to maintain appropriate levels of functional proteins needed for the altered level of cellular homeostasis in the presence of nicotine. The ubiquitin-proteasome system may accomplish this task by getting rid of denatured proteins as well as acting by as a regulator of other signaling pathways and/or through regulating the density of receptors such as the nAChRs (Fig. 6).

In this study, 3 and 14 days of nicotine administration were used to study the subacute and chronic effects of nicotine, respectively. The route of administration was chosen to ensure equal amounts of nicotine or saline given in an identical manner, thereby reducing potential experimental errors in the measured gene expression levels, which were induced by variation among animals. While it does not mimic human smoking exactly, this protocol does reproduce the cyclical levels of plasma nicotine concentration observed in smokers between waking and sleeping hours $[2,3,36]$. By using an initiation phase for habituation and an appropriate saline-control group, we believe the stress of ip injection has been taken into account, which has been documented in the c-fos expression study [47]. Indeed, this dose and method of administration have been utilized effectively in a number of research laboratories (e.g., see Refs. [2,3,14,36,40]).

While there was upregulation of many ubiquitin genes in the MBH after 3 days of nicotine administration, in general, the ubiquitin-proteasomal gene families demonstrated coregulation in the MBH and PFC after 14 days. This suggests that a chronic effect of nicotine is required within these brain regions before the ubiquitin-proteasomal gene families are regulated. Whether the increase in expression of ubiquitins $\mathrm{B}$ and $\mathrm{C}$ and other members of the ubiquitin and proteasome families in the PFC and $\mathrm{MBH}$ is the direct result of nicotine's oxidative stress effect or an indirect effect of increased synaptic activity over time is unclear. Further study will be required to decipher the biochemical mechanism(s) by which the ubiquitin-proteasome pathway is activated in response to nicotine.

While SUMO activation and substrate targeting has analogy with the ubiquitin pathway, the final destination of sumoylated proteins is not the $26 \mathrm{~S}$ proteasome. SUMO proteins often compete with ubiquitin for the same substrates, but once sumoylated, the protein may be transferred to the nucleus [27]. From the findings of the present study, the upregulation of both SUMO-1 and SUMO-2, and Ubc9 along with the activation of the ubiquitin system in the PFC at day 14 of nicotine exposure strongly suggests that the two pathways are competing for the same protein substrates (Fig. 6). The CCT is a hetero-oligomeric complex

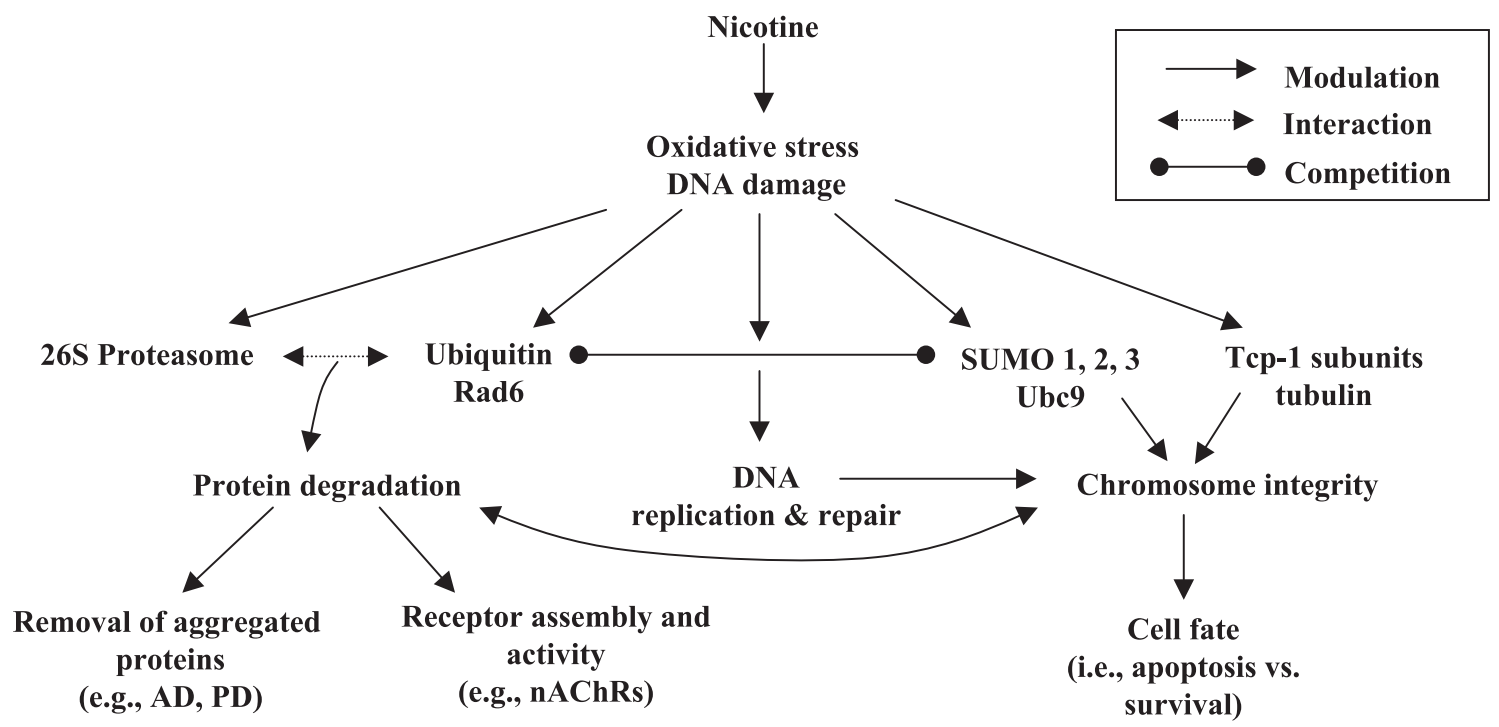

Fig. 6. Proposed model for nicotine's effects on protein modification and degradation pathways. Nicotine administration produces oxidative stress and may cause DNA damage. This effect may modulate the systems (both DNA and protein) responsible for maintaining homeostasis within the cell. These include the ubiquitin/proteasomal system involved in protein degradation, SUMOs and ubiquitin in DNA repair, and the chaperonin system that aids in protein folding. The result is the potential regulation of signaling pathways determining chromosomal integrity as well as the fate of aggregated or key signal proteins. Involvement of SUMO/Ubc9 in connection with ubiquitin/RAD6 and/or chaperonin systems may ultimately play a role in the survival signals in cells (e.g., neurons) in response to nicotine. 
made up of eight highly conserved subunits regulated in concert to maintain a constant ratio so that the complex is best formed in a fixed-subunit composition [32,33]. An increase of three CCT subunits in the PFC and a decrease of four CCT subunits in the $\mathrm{MBH}$ support the idea of coordinate regulation of $\mathrm{CCT}$ subunits at the transcription level. Furthermore, protein degradation of CCT is controlled by the ubiquitin-proteasome system [58]. In the PFC, the proteasome system may degrade CCT subunits to force upregulation of CCT gene transcription in order to keep pace with the demands of the cell. It seems that these two pathways are working jointly to promote protein homeostasis in a cell under oxidative stress.

Previous studies also have suggested that nicotine, directly or indirectly, modulates the amount of DNA repair and/or DNA damage tolerance $[30,44,46]$. For example, nicotine increases the repair of plasmid DNA damaged by free radicals in the presence of whole-cell protein extracts from adult myocytes [30]. The authors postulated that nicotine enhances DNA degradation through increasing nuclease activity, which allows more efficient incorporation of nucleotides needed to repair the damaged DNA [30]. On the other hand, a recent study suggested nicotine has protective effects against DNA-damaging agents in head and neck cancer cell lines through a decrease in the activity of stress-activated protein kinase signaling (SAPK/JNK) [44]. In the presence of nicotine, it is likely that the DNA repair machinery is forced to cope with damage resulting from production of reactive oxygen species $[25,26]$. Nevertheless, the exact molecular mechanisms underlying nicotine's effect on DNA repair and DNA damage tolerance remain largely unknown and controversial. Recent evidence strongly implicates ubiquitination and sumoylation in DNA replication/repair and damage tolerance [22,50].

In this context, the SUMO/Ubc9 pathway has been shown to compete with the ubiquitin/RAD6 pathway in fine-tuning the extent to which the cell practices DNA repair. Furthermore, DNA repair is essential in maintaining chromosome integrity and attaining an appropriate cell fate (apoptosis vs. survival). Recent studies indicate that the ubiquitin/proteasome pathway, as well as sumoylation and modification of tubulin by the CCT complex, contributes to the maintenance of chromosomal integrity $[1,5,23,49,53]$. According to the proposed model illustrated in Fig. 6, we hypothesize that the observed increase in the PFC of expression of mRNA for both the ubiquitin/RAD6 and SUMO/Ubc9 genes after nicotine exposure may be indicative of a sustained response to an increased rate of DNA damage induced by oxidative stress. Accordingly, the degree of competition between the SUMO/Ubc9 and ubiquitin/RAD6 pathways in maintaining chromosomal integrity should correlate with the mode of nicotine-induced stress response eventually leading to alterations in cell fate. This model provides an intriguing mechanism by which nicotine may prevent or delay apoptosis, although chromosomal integrity is compromised (Fig. 6). However, future studies are needed to address the molecular mechanisms by which nicotine induces DNA damage and the types of linkage ubiquitin molecules form after their nicotineinduced expression.

Although this model correlates with the findings in the PFC, it may seem to contradict the effects we observed in the $\mathrm{MBH}$ at 14 days, i.e., the downregulation of ubiquitin/ proteasome and CCT pathways. One of the explanations for the differential response of brain regions to nicotine may be compositional differences of the nAChRs. This diversity in different parts of the brain results in relatively different sensitization/desensitization profiles for each region and even nuclei. Therefore, it is possible that the periods of treatment (e.g., 3 or 14 days) translate into different exposure periods for the cells in the PFC vs. the $\mathrm{MBH}$. This asynchrony also may be reflected in the transcriptional response in the PFC and $\mathrm{MBH}$ to nicotine. Furthermore, the time required for adaptation to a nicotine-altered cellular environment may differ among brain regions, resulting in differential needs for the maintenance of cellular homeostasis, hence leading to alternative cell fates.

Finally, an inverse association has been reported between the prevalence of tobacco smoking and the occurrence of Alzheimer's and Parkinson's diseases [42]. Both of these diseases have been linked to proteasomal and CCT deregulation [38,41]. We suggest a possible mechanism for the inverse association with nicotine is through its effects on the ubiquitin-proteasomal and CCT pathways (Fig. 6). Future studies focusing on how and why nicotine is affecting these gene families hold promise for enhancing our understanding of the observed inverse association between nicotine usage and these neurodegenerative diseases, as well as for providing novel mechanisms through which neurons may be adapting to the actions of nicotine.

\section{Acknowledgement}

This project was in part supported by National Institute of Health grants R01 DA-13783 and DA-12844 to MDL.

\section{References}

[1] C. Agueli, F. Geraci, G. Giudice, L. Chimenti, D. Cascino, G. Sconzo, A constitutive $70 \mathrm{kDa}$ heat-shock protein is localized on the fibres of spindles and asters at metaphase in an ATP-dependent manner: a new chaperone role is proposed, Biochem. J. 360 (2001) 413-419.

[2] L. Bellinger, A. Cepeda-Benito, R.L. Bullard, P.J. Wellman, Effect of i.c.v. infusion of the alpha-MSH agonist MTII on meal patterns in male rats following nicotine withdrawal, Life Sci. 73 (2003) $1861-1872$.

[3] L. Bellinger, A. Cepeda-Benito, P.J. Wellman, Meal patterns in male rats during and after intermittent nicotine administration, Pharmacol. Biochem. Behav. 74 (2003) 495-504.

[4] S.V. Bhagwat, C. Vijayasarathy, H. Raza, J. Mullick, N.G. Avadhani, Preferential effects of nicotine and 4-( $N$-methyl- $N$-nitrosamine $)-1-(3-$ pyridyl)-1-butanone on mitochondrial glutathione $S$-transferase A4-4 
induction and increased oxidative stress in the rat brain, Biochem. Pharmacol. 56 (1998) 831-839.

[5] S. Biggins, N. Bhalla, A. Chang, D.L. Smith, A.W. Murray, Genes involved in sister chromatid separation and segregation in the budding yeast Saccharomyces cerevisiae, Genetics 159 (2001) 453-470.

[6] P. Bonaventure, H. Guo, B. Tian, X. Liu, A. Bittner, B. Roland, R. Salunga, X.J. Ma, F. Kamme, B. Meurers, M. Bakker, M. Jurzak, J.E. Leysen, M.G. Erlander, Nuclei and subnuclei gene expression profiling in mammalian brain, Brain Res. 943 (2002) 38-47.

[7] L.J. Born, K.K. Kharbanda, D.L. McVicker, R.K. Zetterman, T.M. Donohue Jr., Effects of ethanol administration on components of the ubiquitin proteolytic pathway in rat liver, Hepatology 23 (1996) $1556-1563$.

[8] S.A. Bustin, Absolute quantification of mRNA using real-time reverse transcription polymerase chain reaction assays, J. Mol. Endocrinol. 25 (2000) $169-193$.

[9] S.R. Chakrabarti, R. Sood, S. Nandi, G. Nucifora, Posttranslational modification of TEL and TEL/AML1 by SUMO-1 and cell-cycledependent assembly into nuclear bodies, Proc. Natl. Acad. Sci. U. S. A. 97 (2000) 13281-13285.

[10] M. Chrysanthou-Piterou, M.R. Issidorides, Distribution of ubiquitin immunoreactivity in dopamine neurons of chronic alcoholics, Neurol. Res. 21 (1999) 426-432.

[11] A. Ciechanover, The ubiquitin-proteasome pathway: on protein death and cell life, EMBO J. 17 (1998) 7151-7160.

[12] A. Ciechanover, A.L. Schwartz, The ubiquitin-proteasome pathway: the complexity and myriad functions of proteins death, Proc. Natl. Acad. Sci. U. S. A. 95 (1998) 2727-2730.

[13] R.C. Conaway, C.S. Brower, J.W. Conaway, Emerging roles of ubiquitin in transcription regulation, Science 296 (2002) 1254-1258.

[14] V. Di Matteo, M. Pierucci, E. Esposito, Selective stimulation of serotonin $2 \mathrm{c}$ receptors blocks the enhancement of striatal and accumbal dopamine release induced by nicotine administration, J. Neurochem. 89 (2004) 418-429.

[15] T.M. Donohue Jr., The ubiquitin-proteasome system and its role in ethanol-induced disorders, Addict. Biol. 7 (2002) 15-28.

[16] T. Dunckley, R.J. Lukas, Nicotine modulates the expression of a diverse set of genes in the neuronal SH-SY5Y cell line, J. Biol. Chem. 278 (2003) $15633-15640$.

[17] M.D. Ehlers, Activity level controls postsynaptic composition and signaling via the ubiquitin-proteasome system, Nat. Neurosci. 6 (2003) 231-242.

[18] A.J. Fornace Jr., I. Alamo Jr., M.C. Hollander, E. Lamoreaux, Ubiquitin mRNA is a major stress-induced transcript in mammalian cells, Nucleic Acids Res. 17 (1989) 1215-1230.

[19] F.U. Hartl, M. Hayer-Hartl, Molecular chaperones in the cytosol: from nascent chain to folded protein, Science 295 (2002) 1852-1858.

[20] T. Hayashi, M. Noga, M. Matsuda, Nucleotide sequence and expression of the rat polyubiquitin mRNA, Biochim. Biophys. Acta 1218 (1994) $232-234$.

[21] A. Hershko, A. Ciechanover, The ubiquitin system, Annu. Rev. Biochem. 67 (1998) 425-479.

[22] C. Hoege, B. Pfander, G.L. Moldovan, G. Pyrowolakis, S. Jentsch, RAD6-dependent DNA repair is linked to modification of PCNA by ubiquitin and SUMO, Nature 419 (2002) 135-141.

[23] J. Joseph, S.H. Tan, T.S. Karpova, J.G. McNally, M. Dasso, SUMO-1 targets RanGAP1 to kinetochores and mitotic spindles, J. Cell Biol. 156 (2002) 595-602.

[24] J.K. Kane, S.L. Parker, S.G. Matta, Y. Fu, B.M. Sharp, M.D. Li, Nicotine up-regulates expression of orexin and its receptors in rat brain, Endocrinology 141 (2000) 3623-3629.

[25] S.H. Keller, J. Lindstrom, P. Taylor, Inhibition of glucose trimming with castanospermine reduces calnexin association and promotes proteasome degradation of the alpha-subunit of the nicotinic acetylcholine receptor, J. Biol. Chem. 273 (1998) 17064-17072.

[26] S.H. Keller, J. Lindstrom, M. Ellisman, P. Taylor, Adjacent basic amino acid residues recognized by the COP I complex and ubiquitination govern endoplasmic reticulum to cell surface trafficking of the nicotinic acetylcholine receptor alpha-subunit, J. Biol. Chem. 276 (2001) 18384-18391.

[27] K.I. Kim, S.H. Baek, C.H. Chung, Versatile protein tag, SUMO: its enzymology and biological function, J. Cell. Physiol. 191 (2002) $257-268$.

[28] O. Konu, J.K. Kane, T. Barrett, M.P. Vawter, R. Chang, J.Z. Ma, D.M. Donovan, B. Sharp, K.G. Becker, M.D. Li, Region-specific transcriptional response to chronic nicotine in rat brain, Brain Res. 909 (2001) $194-203$

[29] O. Konu, X. Xu, J.Z. Ma, J. Kane, J. Wang, S.J. Shi, M.D. Li, Application of a customized pathway-focused microarray for gene expression profiling of cellular homeostasis upon exposure to nicotine in PC12 cells, Brain Res. Mol. Brain Res. 121 (2004) 102-113.

[30] P.L. Kozlovskis-Wade, M.J. Smets, R.J. Myerburg, The effect of nicotine on DNA repair in adult myocytes, J. Mol. Cell. Cardiol. 30 (1998) 1483-1491.

[31] H. Kubota, G. Hynes, K. Willison, The chaperonin containing tcomplex polypeptide 1 (TCP-1). Multisubunit machinery assisting in protein folding and assembly in the eukaryotic cytosol, Eur. J. Biochem. 230 (1995) 3-16.

[32] H. Kubota, S. Matsumoto, S. Yokota, H. Yanagi, T. Yura, Transcriptional activation of mouse cytosolic chaperonin CCT subunit genes by heat shock factors HSF1 and HSF2, FEBS Lett. 461 (1999) 125-129.

[33] H. Kubota, S. Yokota, H. Yanagi, T. Yura, Structures and co-regulated expression of the genes encoding mouse cytosolic chaperonin CCT subunits, Eur. J. Biochem. 262 (1999) 492-500.

[34] M.D. Li, J.K. Kane, J. Wang, J.Z. Ma, Time-dependent changes in transcriptional profiles within five rat brain regions in response to nicotine treatment, Mol. Brain Res. 132 (2004) 168-180 this issue.

[35] M.D. Li, G.J. MacDonald, J.J. Ford, Breed differences in expression of inhibin/activin subunits in porcine anterior pituitary glands, Endocrinology 138 (1997) 712-718.

[36] M.D. Li, J.K. Kane, S.L. Parker, K. McAllen, S.G. Matta, B.M. Sharp, Nicotine administration enhances NPY expression in the rat hypothalamus, Brain Res. 867 (2000) 157-164.

[37] M.D. Li, O. Konu, J.K. Kane, K.G. Becker, Microarray technology and its application on nicotine research, Mol. Neurobiol. 25 (2002) $265-285$.

[38] M. Lopez Salon, L. Morelli, E.M. Castano, E.F. Soto, J.M. Pasquini, Defective ubiquitination of cerebral proteins in Alzheimer's disease, J. Neurosci. Res. 62 (2000) 302-310.

[39] R. Mahajan, C. Delphin, T. Guan, L. Gerace, F. Melchior, A small ubiquitin-related polypeptide involved in targeting RanGAP1 to nuclear pore complex protein RanBP2, Cell 88 (1997) 97-107.

[40] D.H. Malin, J.R. Lake, P. Newlin-Maultsby, L.K. Roberts, J.G. Lanier, V.A. Carter, J.S. Cunningham, O.B. Wilson, Rodent model of nicotine abstinence syndrome, Pharmacol. Biochem. Behav. 43 (1992) 779-784

[41] K.S. McNaught, P. Jenner, Proteasomal function is impaired in substantia nigra in Parkinson's disease, Neurosci. Lett. 297 (2001) $191-194$.

[42] S. Mihailescu, R. Drucker-Colin, Nicotine, brain nicotinic receptors, and neuropsychiatric disorders, Arch. Med. Res. 31 (2000) $131-144$.

[43] A. Minty, X. Dumont, M. Kaghad, D. Caput, Covalent modification of p73alpha by SUMO-1. Two-hybrid screening with p73 identifies novel SUMO-1-interacting proteins and a SUMO-1 interaction motif, J. Biol. Chem. 275 (2000) 36316-36323.

[44] N. Onoda, A. Nehmi, D. Weiner, S. Mujumdar, R. Christen, G. Los, Nicotine affects the signaling of the death pathway, reducing the response of head and neck cancer cell lines to DNA damaging agents, Head Neck 23 (2001) 860-870.

[45] G. Paxinos, C. Watson, The Rat Brain in Stereotaxic Coordinates, XXVI edn., Academic Press, Sydney, 1986, 237 pp.

[46] G.P. Pfeifer, M.F. Denissenko, M. Olivier, N. Tretyakova, S.S. Hecht, P. Hainaut, Tobacco smoke carcinogens, DNA damage and 
p53 mutations in smoking-associated cancers, Oncogene 21 (2002) $7435-7451$.

[47] A.E. Ryabinin, Y.M. Wang, D.A. Finn, Different levels of Fos immunoreactivity after repeated handling and injection stress in two inbred strains of mice, Pharmacol. Biochem. Behav. 63 (1999) 143-151.

[48] H. Saitoh, J. Hinchey, Functional heterogeneity of small ubiquitinrelated protein modifiers SUMO-1 versus SUMO-2/3, J. Biol. Chem. 275 (2000) 6252-6258.

[49] M.P. Shekhar, A. Lyakhovich, D.W. Visscher, H. Heng, N. Kondrat, Rad6 overexpression induces multinucleation, centrosome amplification, abnormal mitosis, aneuploidy, and transformation, Cancer Res. 62 (2002) 2115-2124.

[50] P. Stelter, H.D. Ulrich, Control of spontaneous and damage-induced mutagenesis by SUMO and ubiquitin conjugation, Nature 425 (2003) 188- 191 .

[51] H. Sternlicht, G.W. Farr, M.L. Sternlicht, J.K. Driscoll, K. Willison, M.B. Yaffe, The t-complex polypeptide 1 complex is a chaperonin for tubulin and actin in vivo, Proc. Natl. Acad. Sci. U. S. A. 90 (1993) 9422-9426.

[52] T.S. Tanaka, S.A. Jaradat, M.K. Lim, G.J. Kargul, X. Wang, M.J. Grahovac, S. Pantano, Y. Sano, Y. Piao, R. Nagaraja, H. Doi, W.H. Wood III, K.G. Becker, M.S. Ko, Genome-wide expression profiling of mid-gestation placenta and embryo using a 15,000 mouse developmental cDNA microarray, Proc. Natl. Acad. Sci. U. S. A. 97 (2000) 9127-9132.

[53] H.D. Ulrich, Degradation or maintenance: actions of the ubiquitin system on eukaryotic chromatin, Eukaryot. Cell 1 (2002) 1-10.

[54] R.N. Van Gelder, M.E. von Zastrow, A. Yool, W.C. Dement, J.D. Barchas, J.H. Eberwine, Amplified RNA synthesized from limited quantities of heterogeneous cDNA, Proc. Natl. Acad. Sci. U. S. A. 87 (1990) 1663-1667.

[55] D. Voges, P. Zwickl, W. Baumeister, The 26S proteasome: a molecular machine designed for controlled proteolysis, Annu. Rev. Biochem. 68 (1999) 1015-1068.

[56] D. Yildiz, N. Ercal, D.W. Armstrong, Nicotine enantiomers and oxidative stress, Toxicology 130 (1998) 155-165.

[57] D. Yildiz, Y.S. Liu, N. Ercal, D.W. Armstrong, Comparison of pure nicotine- and smokeless tobacco extract-induced toxicities and oxidative stress, Arch. Environ Contam. Toxicol. 37 (1999) 434-439.

[58] S. Yokota, T. Kayano, T. Ohta, M. Kurimoto, H. Yanagi, T. Yura, H. Kubota, Proteasome-dependent degradation of cytosolic chaperonin CCT, Biochem. Biophys. Res. Commun. 279 (2000) 712-717.

[59] H. Yue, P.S. Eastman, B.B. Wang, J. Minor, M.H. Doctolero, R.L. Nuttall, R. Stack, J.W. Becker, J.R. Montgomery, M. Vainer, R. Johnston, An evaluation of the performance of cDNA microarrays for detecting changes in global mRNA expression, Nucleic Acids Res. 29 (2001) E41-1. 